\title{
Trends in hospitalization, mortality, and timing of colonoscopy in patients with acute lower gastrointestinal bleeding
}

\section{(ㄷ)(요 $\odot$}

\section{Authors}

Kalpit Devani ${ }^{1}$, Dhruvil Radadiya², Paris Charilaou ${ }^{3}$, Tyler Aasen ${ }^{4}$, Chakradhar M. Reddy ${ }^{4}$, Mark Young ${ }^{4}$, Bhaumik Brahmbhatt ${ }^{5}$, Don C. Rockey ${ }^{6}$

Institutions

1 Division of Gastroenterology and Liver Disease, Department of Internal Medicine, Prisma Health, University of South Carolina, Greenville, South Carolina, United States

2 Department of Internal Medicine, East Tennessee State University, Johnson City, Tennessee, United States

3 Division of Gastroenterology \& Hepatology, Department of Internal Medicine, Saint Peter's University Hospital/ Rutgers - Robert Wood Johnson Medical School, New Brunswick, New Jersey, United States

4 Division of Gastroenterology \& Hepatology, Department of Internal Medicine, East Tennessee State University, Johnson City, Tennessee, United States

5 Division of Gastroenterology \& Hepatology, Department of Internal Medicine, Mayo Clinic, Jacksonville, Florida, United States

6 Division of Gastroenterology, Department of Internal Medicine, Medical University of South Carolina, United States

submitted 29.7.2020

accepted after revision 9.12.2020

\section{Bibliography}

Endosc Int Open 2021; 09: E777-E789

DOI 10.1055/a-1352-3204

ISSN 2364-3722

(c) 2021. The Author(s).

This is an open access article published by Thieme under the terms of the Creative Commons Attribution-NonDerivative-NonCommercial License, permitting copying and reproduction so long as the original work is given appropriate credit. Contents may not be used for commercial purposes, or adapted, remixed, transformed or built upon. (https://creativecommons.org/licenses/by-nc-nd/4.0/)

Georg Thieme Verlag KG, Rüdigerstraße 14,

70469 Stuttgart, Germany

Corresponding author

Kalpit Devani, MD, Gastroenterology \& Liver Center, 890 West Faris Road, Suite 100, Greenville, SC 29605, United States Fax: +1-423-439-6387

Kalpit_devani@yahoo.com $\bigoplus$ Supplementary material is available under
https://doi.org/10.1055/a-1352-3204

\section{ABSTRACT}

Background and study aims Current guidelines conditionally recommend performing early colonoscopy (EC) (<24 hours) in patients admitted with acute lower gastrointestinal bleeding (LGIB). It remains unclear whether this practice is implemented widely. Therefore, we used the Nationwide Inpatient Sample to investigate trends for timing of colonoscopy in patients admitted with acute LGIB. We also assessed trend of hospitalization and mortality in patients with LGIB.

Patients and methods Adult patients with LGIB admitted from 2005 to 2014 were examined. ICD-9-CM codes were used to extract LGIB discharges. Trends were assessed using Cochrane-Armitage test. Factors associated with mortality, cost of hospitalization, and length of stay (LOS) were assessed by multivariable mixed-effects and exact-matched logistic, linear regression, and accelerated-failure time models, respectively.

Results A total of 814,647 patients with LGIB were included. The most common etiology of LGIB was diverticular bleeding (49\%) and $45 \%$ of patients underwent EC. Over the study period, there was no change in the trend of colonoscopy timing. Although admission with LGIB increased over the study period, the mortality rate decreased for patients undergoing colonoscopy. Independent predictors of mortality were age, surgery (colostomy/colectomy) during admission, intensive care unit admission, acute kidney injury, and blood transfusion requirement. Timing of colonoscopy was not associated with mortality benefit. However, cost of hospitalization was \$1,946 lower and LOS was 1.6 days shorter with EC.

Conclusion Trends in colonoscopy timing in management of LGIB have not changed over the years. EC is associated with lower LOS and cost of hospitalization but it does not appear to improve inpatient mortality. 


\section{Introduction}

Lower gastrointestinal bleeding (LGIB), defined as bleeding originating either from the colon or rectum [1], accounts for substantial morbidity and mortality in the United States [2]. LGIB admissions accounted for approximately 109 per 100,000 emergency department visits in the United States in year 2012 and are on rise [3]. With prevalent use of antithrombotics, LGIB will remain a common gastrointestinal emergency. Although generally considered to have a better prognosis than upper gastrointestinal bleeding (UGIB), it was reported in one study that LGIB was associated with a higher mortality rate, longer length of stay (LOS), and higher healthcare resource utilization compared to UGIB [4].

A cornerstone of LGIB management is colonoscopy, which has both diagnostic and therapeutic potential [5-7]. Although colonoscopy has been accepted to have an important role in LGIB, the optimal timing of it in LGIB is less clear. Currently, American College of Gastroenterology (ACG) guidelines have recently encouraged colonoscopy within 24 hours of presentation for patients with clinical features suggesting high risk [6]. In patients with severe acute LGIB, the American Society for Gastrointestinal Endoscopy (ASGE) has recommended early colonoscopy (EC) within 24 hours of admission following a rapid bowel preparation $[8,9]$. However, the evidence supporting these recommendations remains weak. Most of the previously published studies on the effect of EC on outcomes have been small in sample size or single-center, thus the impact of EC for clinical outcomes other than mortality remains uncertain [1014]. Consequently, the role of EC in LGIB has been the topic of debate in the gastroenterology community with no single consensus regarding its effect on outcomes, and thus, EC has not been adopted as the standard of care at many hospitals.

In this study, we hypothesized that because of improved care for patients with LGIB, mortality is improving. We also aimed to evaluate recent real-world trends in hospitalization and timing of colonoscopy in patients with LGIB. In addition, we explored factors impacting inpatient mortality, cost of hospitalization, and LOS in this patient population.

\section{Patients and methods}

\section{Design and data source}

We performed a retrospective observational study using the National Inpatient Sample (NIS) [15], which is the largest publicly available database drawn from more than 1000 hospitals in 46 states. It is maintained by the Agency for Healthcare Research and Quality (AHRQ)-sponsored Healthcare Cost and Utilization Project (HCUP). It includes more than 7 million hospital admissions each year, which approximates a $20 \%$ stratified sample of discharges from US community hospitals (nonfederal, short-term, general, and specialty). With weight adjustment, it estimates more than 35 million hospitalizations nationally. Each index hospitalization is de-identified and maintained in the NIS with a unique identifier, principal and secondary discharge diagnoses (up to 25), procedures (up to 15) along with demographic details including age, sex, race, insurance status and type, comorbidities, inpatient mortality, length of stay (LOS), cost of hospitalization and other hospital characteristics. NIS data have been widely used to study trends and predictors of health care usage, procedural adverse effects, cost, quality, and outcomes.

The NIS maintains internal validity in its database with annual data quality assessments, while the external validity of the NIS is supported by comparisons against the following data sources: The American Hospital Association Annual Survey Database, the National Hospital Discharge Survey from the National Center for Health Statistics and the MedPAR (Medicare Provider and Analysis Review) inpatient data from the Centers for Medicare and Medicaid Services.

\section{Population selection}

We used a previously published algorithm to identify patients with LGIB using principle and secondary discharge diagnoses $[15,16]$. Patients with a specific International Classification of Diseases, Ninth Revision, Clinical Modification (ICD-9-CM) code for acute LGIB as principal discharge diagnosis or secondary diagnosis of potential source of acute LGIB with concomitant principal diagnosis of unspecified LGIB were identified from the NIS database from the years 2005 through 2014. The principal diagnosis was defined as the diagnosis determined to be the principal reason for hospitalization. Diagnoses that were considered to be specific for acute LGIB were: diverticulosis or diverticulitis of the colon with hemorrhage, Dieulafoy lesion, internal, external or unspecified hemorrhoids with bleeding, angiodysplasia of the intestine with hemorrhage, and hemorrhage of the rectum and anus. We also included patients with a potential source of acute LGIB (malignant neoplasm of the colon, rectum, rectosigmoid junction; benign neoplasm of colon or rectum, intestinal ischemia, non-infectious colitis, inflammatory bowel disease, infectious enterocolitis, colon ulcer, solitary rectal ulcer syndrome, vascular injury of the intestine, and anal fissure), while having a concomitant principal diagnosis of "unspecified LGIB." ICD 9-CM codes used to identify principal and secondary discharge diagnoses are shown in Supplementary Table 1. Patients less than 18 years old and those missing the following data were excluded: age, sex, or inpatient mortality. Admissions that were marked as "elective," did not receive a colonoscopy, or those who had concomitantly documented UGIB were also excluded. Cases without colonoscopy were excluded as the question in our study was the timing of the colonoscopy and not whether it was done or not; in that way, the patients we selected were, in retrospect, stable and safe enough for them to undergo a colonoscopy during the admission. Performance of colonoscopy during admission was determined by querying all procedural codes for the ICD-9-CM codes corresponding to colonoscopy (Supplementary Table 1). EC was defined as colonoscopy performed within 24 hours of admission (PRDAY 0-1) and late colonoscopy was defined as having colonoscopy performed after 24 hours of admission (PRDAY $>1$ ). Subsequently, cases with invalid or missing data on procedure days were excluded. Of note, Procedure Day is one of most accurate variable in NIS and represents the day of the procedure rather than the day the procedure was ordered or the 
claim was submitted [17]. - Fig. 1 summarizes the cohort selection. For sensitivity analysis, performance of esophagogastroduodenoscopy were identified using codes delineated in Supplementary Table 2. Institutional Review Board approval was not required as NIS is ade - identified database available to the public.

\section{Variables and outcomes}

Each index hospitalization included patient demographics such as age, sex, race, and insurance status. Hospital characteristics such as urban vs rural location, teaching status of the hospital, hospital bed size, and hospital region (Northeast, Midwest, South, or West Northeast, Midwest, South, or West) were also available. Insurance status was categorized as Medicare, Medicaid, private insurance, and other. Utilization of invasive support/monitoring that conventionally involves an intensive care unit (ICU) setting was used as a surrogate to identify ICU admission (Supplementary Table 3). Use of blood transfusion during hospitalization was identified using ICD-9 CM codes (Supplementary Table 3). We used the Charlson Comorbidity Index $(\mathrm{CCl})$ to define the overall severity of comorbidities, because comorbid conditions are known to negatively influence outcomes of the hospitalization. The $\mathrm{CCl}$ is a widely-used index to measure the severity of co-morbidity burden from administrative databases $[18,19]$. The $\mathrm{CCl}$ was calculated for each admission as described in Supplementary Table4. Hospitalization costs were calculated by multiplying cost-to-charge (CCR) ratios provided by the Health Care Cost and Utilization Project (HCUP) by the TOTCHG (total charges) variable, subsequently weighted for missing values and finally adjusted for annual inflation using inflation calculator [20], with reference to year 2014.

The primary aim of this study was to assess trends for LGIB admission, timing of colonoscopy, and mortality over the study period. The secondary aims were to assess factors affecting inpatient all-cause mortality, cost of hospitalization, and LOS with particular interest in timing of colonoscopy.

\section{Statistical analysis}

We calculated weighted means \pm standard errors (SE) and \% for continuous and categorical variables, respectively, for both the groups: EC and late colonoscopy. Trend analyses were conducted using mixed models (Hospital ID-Year as the random intercept), against unit increase of year. Univariate analysis correlation tables were done to establish significant associations between each covariate and the outcomes of inpatient mortality, hospitalization costs and LOS, using mixed-effects logistic regression, mixed-effects log-transformed linear regression and mixed-effects accelerated-failure-time (AFT) with log-normal distribution model, respectively. AFT models can estimate LOS while censoring inpatient deaths, as the event is defined "alive at discharge." An additional benefit of AFT models is the interpretability of the Time Ratio on (geometric mean of) LOS, allowing to compare longer/shorter LOS by $\%$ at the level of each covariate, while not being subject to the proportional hazards assumption. Covariates with clinical merit and statistical significance at $P<0.1$ on univariate analysis have been included in the multivariate models, taking into account multicollinearity, possible interactions, and overfitting. Multicollinearity was assessed by the Variance Inflation Factor (goal VIF<10). The goodness of fit of the logistic regression model was evaluated using the area under the curve (AUC, i. e., c-statistic) and goodness of fit for linear regression (hospitalization costs) was assessed using observed vs fitted plots and pseudo- $R^{2}$. Details of each analysis are shown in their respective multivariable analysis models. Sensitivity analyses for each outcome were done by including the performance and timing of esophagogastroduodenoscopy (EGD) as an interaction term with early and late colonoscopy, therefore adjusting for potential selection bias for early vs. late colonoscopy, which can result from the performance of an EGD itself, its timing, as well as its potential findings.

Acknowledging the retrospective nature of the study and the risk of selection bias for or against undergoing an early vs. late colonoscopy, we performed exact-matching among patients who underwent colonoscopy, taking into account various confounders that could potentially affect the following: 1. the decision of clinicians to choose early vs. late colonoscopy for a patient; and 2. outcomes (inpatient mortality, hospitalization costs and LOS). The exact-matching analysis estimated its own analytical weights according to the total number of matched cases within each matched stratum, therefore the estimated effects are by design not nationally-weighted. Continuous variables were semi-exact-matched, i.e., matching groups were created to facilitate optimal balance between potential residual mismatch (variability) and powering (number of cases) within matched strata; these variables were then additionally included in the post-matching regression models to adjust for any residual variability (i.e. double-robust regression). In addition to the matching variables used, patients were matched to the same hospital within the same year to account for inherent differences between hospitals and endoscopists. Further details on the number of matched observations and number of matched strata, matching variables used, and post-matching regression covariates are as described in multivariable analysis models in results section. The advantage of using exact-matching over other matching methods such as propensity score matching (PSM), is that in contrast to PSM, exact-matching compares patients with exactly the same characteristics (instead of having a similar propensity score potentially reflecting different characteristics), thereby eliminating regression modeling caveats such as multicollinearity, overfitting, miss-specification (i.e. unspecified covariates and/or interactions in the model).

National estimates were calculated using the TRENDWT (trend weight) variable, as recommended by HCUP, while also adjusting for potentially missing hospitals from the LGIB subpopulation. Complex-survey design commands ("svy") were used to account for the NIS complex-survey design, as recommended by HCUP. $P$ values were considered significant a priori at $<0.05$. All analyses were performed with Stata MP 14.2 (Stata-Corp, College Station, Texas, United States). 


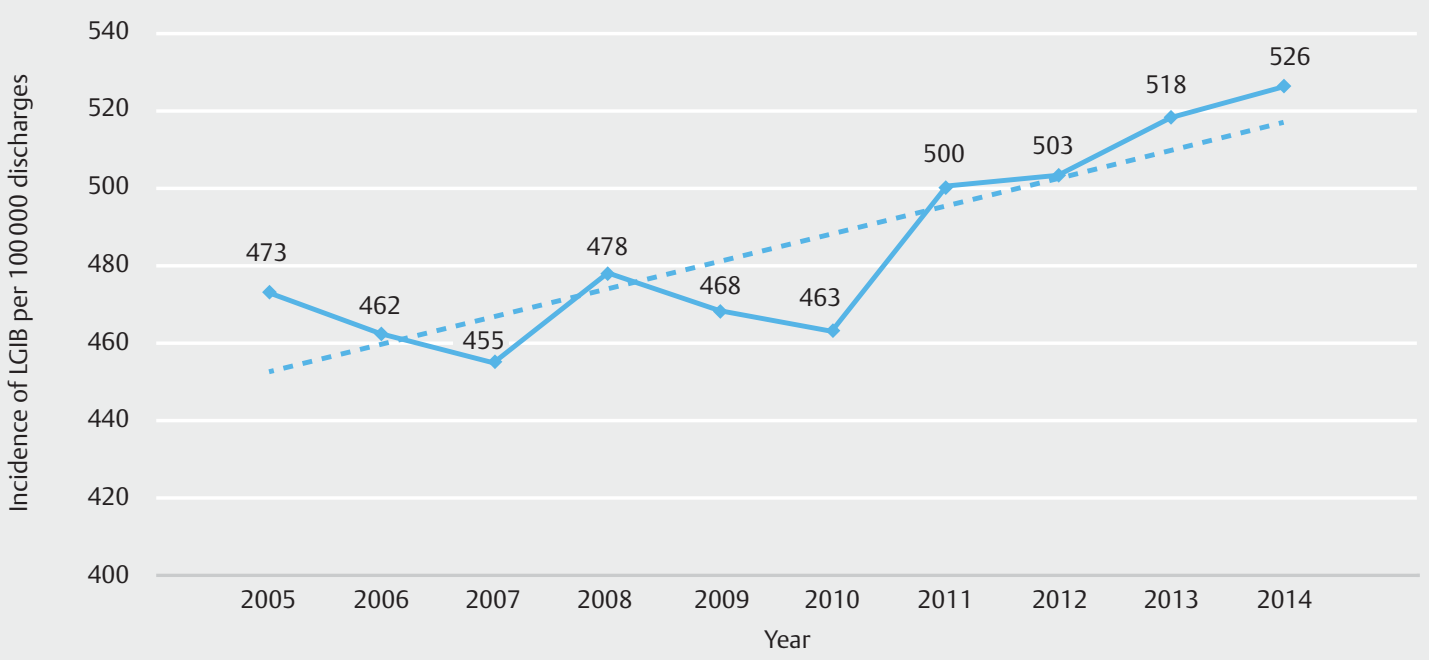

a

$60 \%$

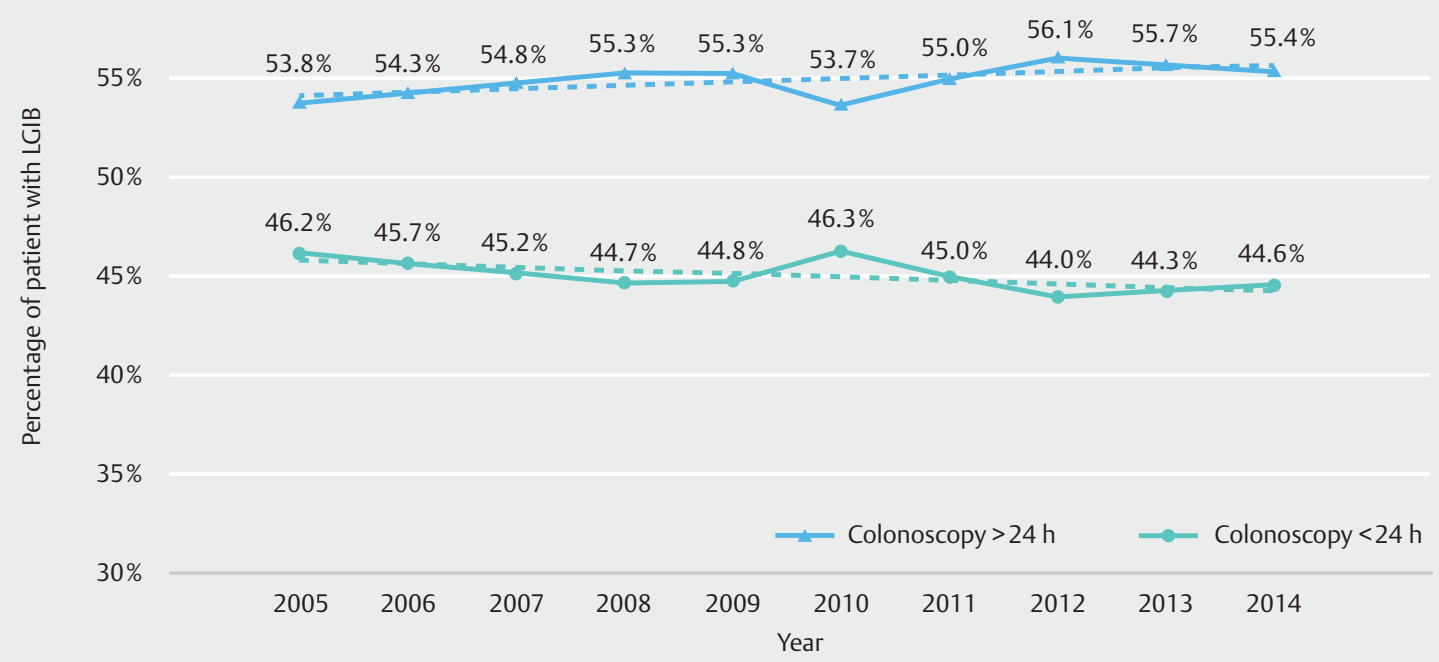

b

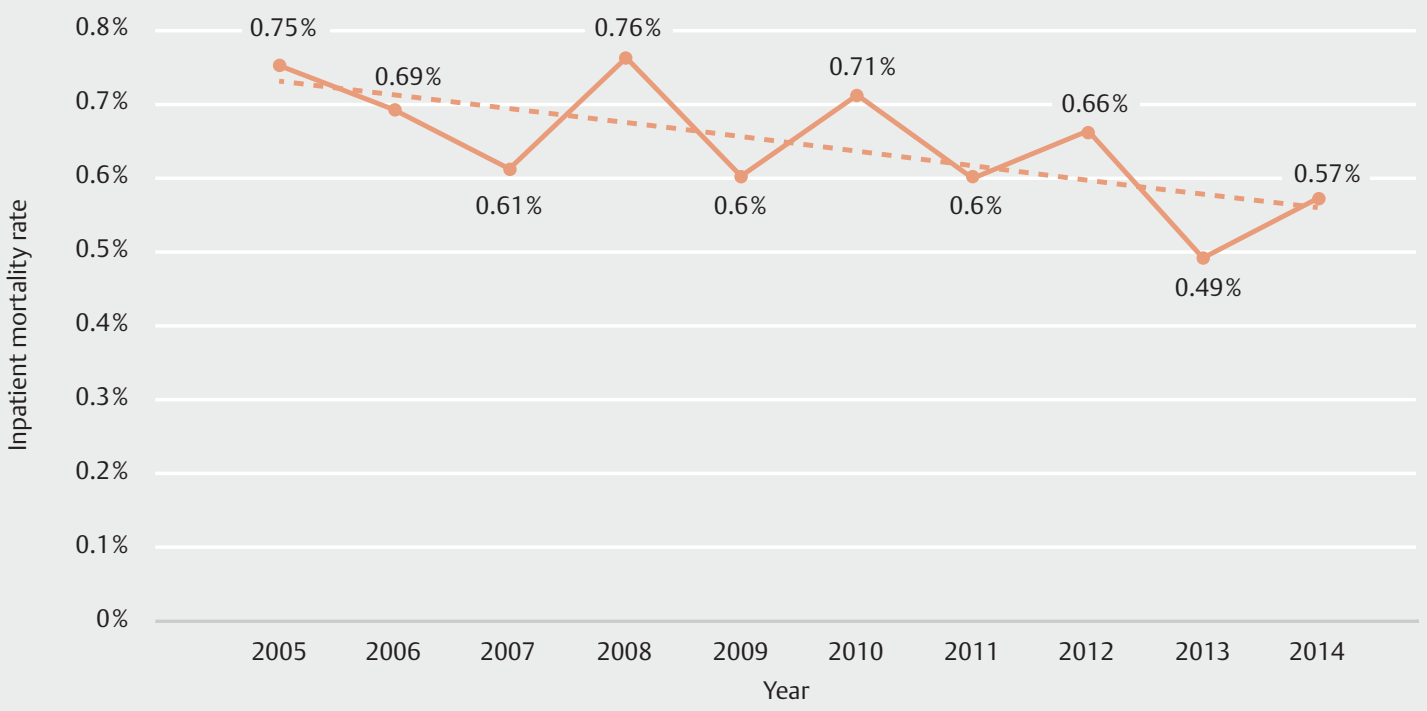

c

- Fig. 1 a Trends for acute lower gastrointestinal bleeding (LGIB). The figure depicts the number of patients with LGIB admitted per 100,000 hospital discharges over the study period from 2005 to 2014 and shows rising incidence of LGIB admissions $(P$ trend $<0.001)$. b Trends for colonoscopy timing among patients with acute lower gastrointestinal bleeding (LGIB). The proportion of patients having early or late colonoscopy over the study period are shown. Both trends of timing are statistically non-significant (early colonoscopy $P$ trend $=0.07$, late colonoscopy $P$ trend $=0.06$ ). $\mathbf{c}$ Trends for inpatient mortality in LGIB over the study period. The rates of inpatient mortality from 2005 to 2014 in the study cohort are shown $(P$ trend $<0.001)$. 


\section{Results}

\section{Baseline characteristics}

A total of 1,643,235 patients with LGIB were identified during the study period and after excluding patients based on exclusion criteria, we included 814,647 patient with LGIB undergoing colonoscopy regardless of timing in the final analysis (Supplementary Fig.1). The mean age of patients in the study population was 72 years, with equal sex distribution, but with a predominance of White patients $(518,395 ; 69 \%)$ as shown in - Table 1. One-fourth of patients $(201,320 ; 25 \%)$ were admitted on a weekend. AKI was present in 66,208 ( $8 \%$ ) of patients. The majority of patients were admitted to urban (733,567; $90 \%)$, large bed size $(493,098 ; 61 \%)$, and teaching hospitals $(357,102 ; 44 \%)$. Southern hospitals accounted for the most patients $(324,537 ; 40 \%)$ of the four regions examined. Although $0.6 \%$ patients $(5,401)$ were admitted to an ICU, $42 \%$ of patients $(344,760)$ had a blood transfusion during hospitalization. The overall mortality rate was $0.6 \%(5,240)$. The mean LOS was 4.5 days, with a cost of hospitalization at $\$ 10,100$. Of note, $46 \%$ $(372,823)$ admitted for LGIB underwent simultaneous EGD.

Forty-five percent of patients $(366,842)$ with LGIB underwent EC while $55 \%(447,805)$ had LC ( $\vee$ Table 1$)$. Patients were slightly older (72 vs. 71 years) and more likely to be female in the LC group [237,823 (53\%) vs. 176,668 (48\%)]. Weekend ad- missions were more common in the LC group $(126,741 ; 28 \%)$ than EC group $(74,579 ; 20 \%)\left(X^{2} P<0.001\right]$. The $C C I$ was slightly higher in the LC group (1.4 vs. 1.1). Patients requiring ICU level care were equally distributed between the two cohorts $(0.6 \%$; 2,867 in LC group and $0.7 \%$; 2,534 in EC group). Blood transfusion was more common in the LC group $(201,633 ; 45 \%$ vs $143,127 ; 39 \%)$. Though the proportion of patients undergoing EGD was higher in the LC than in the EC group (228,372; 51 \% vs $144,451 ; 39 \%)$, stratification based on timing of EGD showed that the LC group had later EGDs (34\%; 150,447 vs. $3 \%$; $11,473)$ while the EC group had more early EGDs (35\%; $127,224$ vs. $16 \% ; 71,706)$. The median LOS was 2 days longer (5.3 vs. 3.5 days) and cost of hospitalization was nearly $\$ 2,000$ higher in LC group $(\$ 11,143$ vs. $\$ 8,817)$. Mortality rates in the two groups was identical $(0.6 \% ; 2,863$ in LC group vs. $0.7 \%$; 2,377 in EC group).

\section{Trends in LGIB admissions, timing of colonoscopy and mortality}

LGIB admissions increased from 472 in 2005 to 526 per 100,000 discharges in year 2014 [ $P$ trend $<0.001)$ ( $>$ Fig. 1a). Of note, the timing of colonoscopy changed very little over the study period (LC changed from $53.8 \%$ to $55.4 \%$; $P$ trend $=0.06$ and EC from $46.2 \%$ to $44.6 \%$; $P$ trend $=0.07)(\triangleright$ Fig. 1 b). The inpati-

- Table 1 Baseline characteristics.

\begin{tabular}{|c|c|c|c|c|c|}
\hline Variables & & $\begin{array}{l}\text { Late colonoscopy } \\
\text { (>24h) } \\
N=447,805(55 \%)\end{array}$ & $\begin{array}{l}\text { Early colonoscopy } \\
(\leq 24 \mathrm{~h}) \\
\mathrm{N}=366,842(45 \%)\end{array}$ & $\begin{array}{l}\text { Total } \\
\mathrm{N}=\mathbf{8 1 4 , 6 4 7}\end{array}$ & $P$ value \\
\hline \multirow[t]{5}{*}{ Patient characteristic } & $\mathrm{Age}^{1}$ & 72 & 71 & 72 & $<0.001$ \\
\hline & Female $^{1}$ & $237,823(53)$ & $176,668(48)$ & $414,491(51)$ & $<0.001$ \\
\hline & Weekend admission ${ }^{1}$ & $126,741(28)$ & $74,579(20)$ & $201,320(25)$ & $<0.001$ \\
\hline & $\mathrm{CCl}\left[\right.$ median; IQR] ${ }^{1}$ & $\begin{array}{l}1.4 \pm 0.1 \\
{[1,0-2]}\end{array}$ & $\begin{array}{l}1.1 \pm 0.1 \\
{[0,0-2]}\end{array}$ & $\begin{array}{l}1.2 \pm 0.1 \\
{[1,0-2]}\end{array}$ & $<0.001^{1}$ \\
\hline & Acute kidney injury ${ }^{1}$ & $43,244(10)$ & $22,963(6)$ & $66,208(8)$ & $<0.001$ \\
\hline \multirow[t]{6}{*}{ Race } & White & $284,980(68)$ & $233,415(69)$ & $518,395(69)$ & \multirow[t]{6}{*}{$<0.001$} \\
\hline & Black & 77,789 (19) & $55,777(17)$ & $133,566(18)$ & \\
\hline & Hispanic & $34,991(8)$ & $28,005(8)$ & $62,996(8)$ & \\
\hline & Asian/Pacific Islander & $8,899(2)$ & $10,669(3)$ & $19,568(3)$ & \\
\hline & Native American & $1,749(0.4)$ & $1,380(0.4)$ & $3,129(0.4)$ & \\
\hline & Other & $9,261(2)$ & $7,620(2)$ & $16,881(2)$ & \\
\hline \multirow[t]{6}{*}{ Insurance type } & Medicare & $329,134(74)$ & $255,182(70)$ & $584,316(72)$ & \multirow[t]{6}{*}{$<0.001$} \\
\hline & Medicaid & $25,686(6)$ & $17,275(5)$ & $42,961(5)$ & \\
\hline & Private & $69,800(16)$ & $74,325(20)$ & $144,124(18)$ & \\
\hline & Self-pay & $13,117(3)$ & $11,587(3)$ & $24,704(3)$ & \\
\hline & No charge & $1,892(0.4)$ & $1,440(0.4)$ & $3,333(0.4)$ & \\
\hline & Other & $7,457(2)$ & $6,606(2)$ & $14,063(2)$ & \\
\hline Hospital location & Urban location & $403,453(90)$ & $330,115(90)$ & $733,567(90)$ & 0.707 \\
\hline
\end{tabular}


- Table 1 (Continuation)

\begin{tabular}{|c|c|c|c|c|c|}
\hline \multicolumn{2}{|l|}{ Variables } & $\begin{array}{l}\text { Late colonoscopy } \\
\text { (>24h) } \\
\mathrm{N}=447,805(55 \%)\end{array}$ & $\begin{array}{l}\text { Early colonoscopy } \\
(\leq 24 \mathrm{~h}) \\
\mathrm{N}=366,842(45 \%)\end{array}$ & $\begin{array}{l}\text { Total } \\
\mathrm{N}=\mathbf{8 1 4 , 6 4 7}\end{array}$ & $P$ value \\
\hline Teaching status & Teaching hospital & $198,701(44)$ & $158,401(43)$ & $357,102(44)$ & 0.016 \\
\hline \multirow[t]{4}{*}{ Hospital region } & Northeast & $125,767(28)$ & 70,593 (19) & $196,359(24)$ & \multirow[t]{4}{*}{$<0.001$} \\
\hline & Midwest & $73,241(16)$ & $71,032(19)$ & $144,273(18)$ & \\
\hline & South & $184,921(41)$ & $139,617(38)$ & $324,537(40)$ & \\
\hline & West & $63,877(14)$ & $85,601(23)$ & $149,477(18)$ & \\
\hline \multirow[t]{3}{*}{ Hospital bed size } & Small & $51,324(12)$ & $46,679(13)$ & $98,003(12)$ & \multirow[t]{3}{*}{$<0.001$} \\
\hline & Medium & $121,583(27)$ & $97,990(27)$ & $219,573(27)$ & \\
\hline & Large & $272,927(61)$ & $220,170(60)$ & $493,098(61)$ & \\
\hline \multirow[t]{14}{*}{ Resource utilization } & ICU Admission & $2,867(0.6)$ & $2,534(0.7)$ & $5,401(0.6)$ & 0.222 \\
\hline & Inpatient mortality & $2,863(0.6)$ & $2,377(0.7)$ & $5,240(0.6)$ & 0.829 \\
\hline & Length of Stay [median] & 4 & 2 & 3 & $<0.001^{2}$ \\
\hline & [Arithmetic mean] & 5.3 & 3.5 & 4.5 & $<0.001$ \\
\hline & [Geometric mean] & 4.3 & 2.7 & 3.6 & $<0.001$ \\
\hline & Cost [median; IQR] & 8,$240 ; 5,745-12,445$ & 6,$093 ; 4,157-9,482$ & 7,$258 ; 4,928-11,255$ & $<0.001^{3}$ \\
\hline & [Arithmetic mean] & 11,143 & 8,817 & 10,100 & $<0.001$ \\
\hline & [Geometric mean] & 8,559 & 6,613 & 7,739 & $<0.001$ \\
\hline & Blood transfusion ${ }^{1}$ & $201,633(45)$ & $143,127(39)$ & $344,760(42)$ & $<0.001$ \\
\hline & Colectomy/colostomy ${ }^{1}$ & $7.973(1.8)$ & $9,558(2.6)$ & $17,531(2.2)$ & $<0.001$ \\
\hline & EGD & $228,372(51)$ & $144,451(39)$ & $372,823(46)$ & $<0.001$ \\
\hline & \multicolumn{5}{|l|}{ EGD timing $^{3}$} \\
\hline & Early EGD & $71,706(16)$ & $127,224(35)$ & $198,930(25)$ & $<0.001$ \\
\hline & Late EGD & $150,447(34)$ & $11,473(3)$ & $161,921(20)$ & $<0.001$ \\
\hline \multicolumn{6}{|c|}{$\begin{array}{l}\text { \% compared with weighted chi-square tests and continuous variables with weighted t-test, unless otherwise specified } \\
\mathrm{Cl} \text {, confidence interval; EGD, esophagogastroduodenoscopy; CCI, Charlson Comorbidity Index; ICU, intensive care unit; IQR, interquartile range. } \\
1 \text { Variables used for exact-matching analysis, which also matched same hospitals within the same year. } \\
2 \text { Tested with generalized linear model with log-transform under Poisson distribution. } \\
{ }^{3} \text { Tested with generalized linear model with log-transform under Normal distribution. }\end{array}$} \\
\hline
\end{tabular}

ent mortality rate decreased slightly from $0.75 \%$ in 2005 to $0.57 \%$ in $2014 P$ trend $<0.001$ ] ( Fig. 1c).

\section{Causes of LGIB}

The most common diagnosis associated with LGIB was diverticular bleeding (49\%; 398,487), followed by anorectal hemorrhage $(26.7 \% ; 217,446)$ and colonic polyps $(11 \% ; 87,192)$ ( $\triangleright$ Table 2). Of secondary etiologies with unspecified LGIB as linked diagnosis, polyps and inflammatory lesion of the colon were most prominent ( $\downarrow$ Table 2 ). It should be noted that some patients had more than one discharge diagnosis code consistent with LGIB or a potential source of LGIB.

\section{Factors associated with inpatient mortality}

There was no statistically significant difference in odds of mortality between the EC and LC group (OR:1.08, $95 \% \mathrm{Cl}: 0.93-$ 1.25, $P=0.336$ ) ( Table 3 ). In addition, results were similar after performing the exact matching (OR:1.77, Cl:0.70-4.50, $P=0.23$ ). We also performed a sensitivity analysis focused on EGD (during the same admission) and found that EGD (whether early or late) did not affect mortality odds compared to no EGD ( $\downarrow$ Table 3). Non-diverticular LGIB had no difference in odds of mortality compared to diverticular LGIB (OR: $0.91, P=0.185$ ). A 10 -year increase in patient age was associated with a $44 \%$ increase in odds of mortality (OR:1.44, P<0.001). The odds of mortality was almost $17 \%$ lower in women than in men (OR:0.83, $P=0.006)$. Weekend admission did not appear to influence the mortality odds compared to weekday admissions 


\begin{tabular}{|c|c|c|c|}
\hline & Late colonoscopy & Early colonoscopy & Total \\
\hline \multicolumn{4}{|l|}{ Primary diagnosis } \\
\hline Diverticular bleeding & $193,938(43.3)$ & $204,549(55.8)$ & $398,487(48.9)$ \\
\hline Anorectal hemorrhage & $119,761(26.8)$ & $97,685(26.5)$ & $217,446(26.7)$ \\
\hline Intestinal arteriovenous malformations & $42,448(9.5)$ & $22,791(6.2)$ & $65,240(8.0)$ \\
\hline Dieulafoy lesion & $1,808(0.4)$ & $1,842(0.5)$ & $3,650(0.4)$ \\
\hline Unspecified LGIB with secondary etiologies & $89,850(20.1)$ & $39,974(10.9)$ & $129,824(15.9)$ \\
\hline Polyps & $63,039(14.1)$ & $24,153(6.6)$ & $87,192(10.7)$ \\
\hline Non-infectious colitis & $8,747(1.9)$ & $4,244(1.2)$ & $12,991(1.6)$ \\
\hline Colonic ulcer & $5,683(1.3)$ & $3,946(1.1)$ & $9,629(1.2)$ \\
\hline Ischemic colitis & $4,387(0.9)$ & $2,713(0.7)$ & $7,100(0.8)$ \\
\hline Inflammatory bowel disease & $3,233(0.7)$ & $2,288(0.6)$ & $5,521(0.7)$ \\
\hline Solitary rectal ulcer & $2,851(0.6)$ & $1,649(0.4)$ & $4,500(0.5)$ \\
\hline Colorectal cancer & $2,148(0.5)$ & $1,296(0.4)$ & $3,443(0.4)$ \\
\hline Gastrointestinal vessel anomaly & $1,227(0.3)$ & $567(0.2)$ & $1,795(0.2)$ \\
\hline Anal fissure & $636(0.1)$ & $408(0.1)$ & $1,043(0.1)$ \\
\hline Infectious colitis & $495(0.1)$ & $365(0.1)$ & $860(0.1)$ \\
\hline
\end{tabular}

(OR:1.12, 95\% Cl: 0.96-1.29, P=0.149). Not surprisingly, increases in the $\mathrm{CCl}$ score of 1 point in patients with LGIB was associated with an increase in odds of mortality by $33 \%$ (OR:1.33, $P<0.001)$. ICU admission and surgery requirement were associated with 18-fold (OR:18.7, $P<0.001)$ and 9-fold rise (OR:9.7, $P<0.001)$ in mortality odds, respectively. Blood transfusion during admission was associated with a $30 \%$ increase in odds of death (OR:1.3; $P<0.001)$. AKI in the setting of LGIB was associated with nearly 3-fold higher mortality odds ( $\triangleright$ Table 3). Compared to Northeast US hospitals, hospitals situated in the Midwest had $28 \%$ lower mortality odds while no difference existed for hospitals located in other parts of the United States. Rural hospitals and teaching hospitals had no difference in mortality odds compared to their counterparts. Moreover, hospital bed size did not affect mortality.

\section{Factors associated with cost of hospitalization}

After adjusting for LOS, the mean cost of hospitalization was approximately $12 \%$ lower in patients who underwent EC (OR:0.88, $95 \%$ Cl: 0.85-0.88, P<0.001) ( Table 4). Here, $12 \%$ represents change over the geometric mean of cost of hospitalization, which corresponds to $\$ 1,946$ reduced cost with EC compared to late colonoscopy. Performance of exact matching yielded similar results (OR: $0.87,95 \% \mathrm{Cl}: 0.85-0.88, P<0.001)$. Sensitivity analysis showed that EGD either early or late minimally impacted cost of hospitalization (cost decrease ranging from $1 \%-7 \%$ ). Per day increase in LOS was associated with $47 \%$ increase in hospitalization cost. Weekend admissions were associated with minimal increase in hospitalization cost
(OR:1.01, P<0.001). AKI, LGIB leading to ICU admission, and the need for surgery or blood transfusion increased cost of hospitalization. Hospitals situated in the Western United States had a $14 \%$ higher cost of hospitalization, while the South and Midwest had $14 \%$ and $2 \%$ lower costs compared to hospitals in the Northeast United States, respectively. Teaching hospitals and urban hospitals had an almost $5 \%$ lower cost of hospitalization compared to rural hospitals. Medium and large bed sizes were associated with lower cost of hospitalization compared to small bed size.

\section{Factors associated with length of stay}

EC was associated with a $32 \%$ shorter LOS compared to patients with late colonoscopy (TR:0.68, 95\% Cl:0.67-0.68, $P<0.001$ ) ( $\triangleright$ Table 5 ). Here, $32 \%$ represents a change over the geometric mean of LOS, which corresponds to a 1.6-day lower LOS with EC compared to late colonoscopy. Further analysis with exact matching revealed similar results (OR:0.64, 95\% Cl:0.63-0.65, $P<0.001)$. Other variables associated with a reduction in LOS included: (1) having an early EGD; (2) male sex; and (3) being located in a rural area ( $\downarrow$ Table 5 ). On the other hand, older age, requiring surgery or a blood transfusion, or developing AKI were associated with a longer LOS ( $>$ Table 5).

\section{Discussion}

We report here that between 2005 and 2014, the number of patients admitted with LGIB has increased, but mortality among these patients is improving. In addition, despite guide- 
- Table 3 Factors associated with inpatient mortality in patients who underwent colonoscopy.

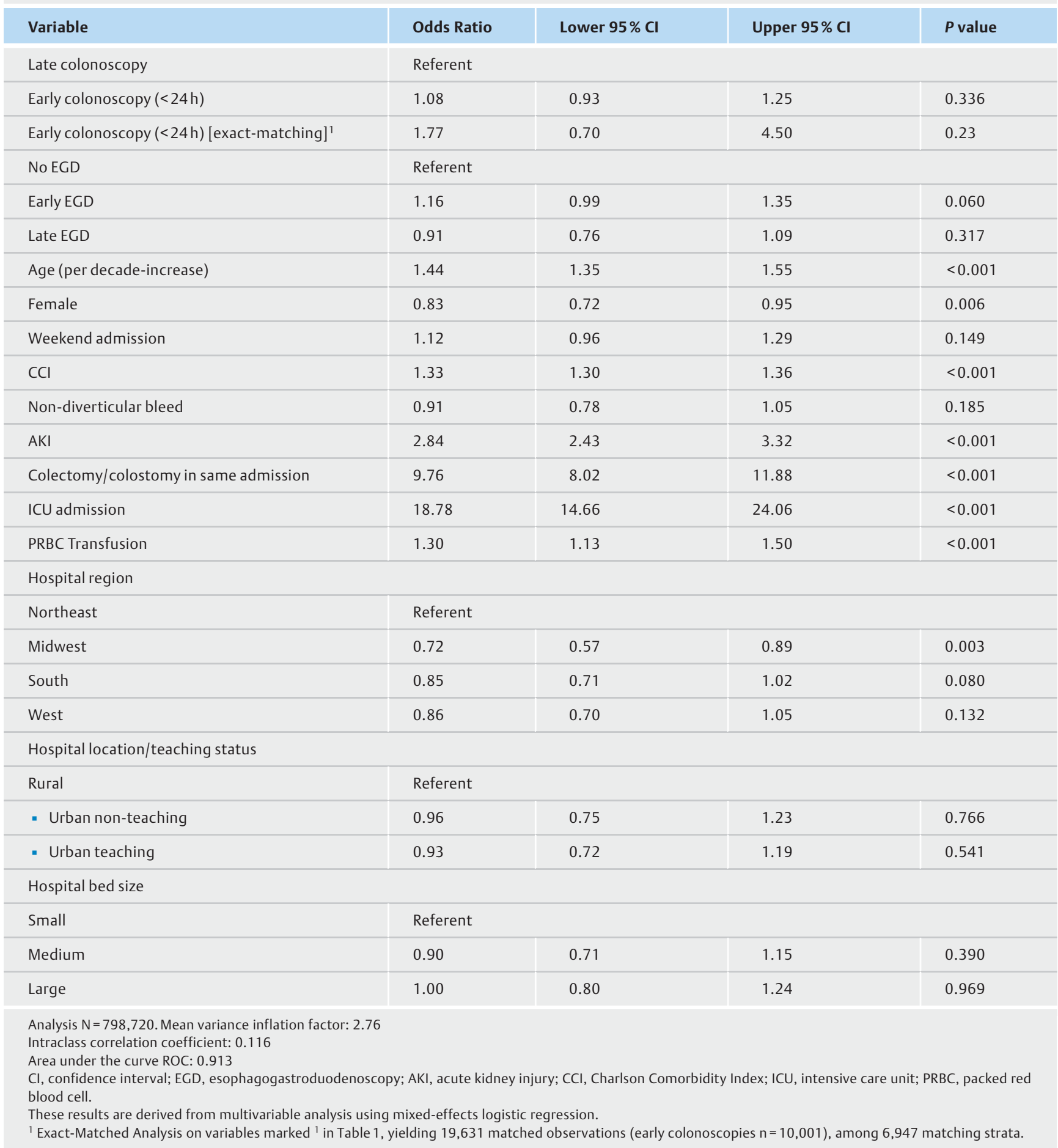

lines and non-randomized data supporting that EC may lead to more rapid identification of colonic lesions [11] and shorter hospital LOS [15, 20-23], EC has not been widely adopted. Finally, in patients admitted with LGIB, EC was associated with decreased LOS and cost of hospitalization, but no significant difference in mortality compared to LC. ICU admission, AKI, and surgery requirement are the strongest predictors of mortality in LGIB.
This study represents a large, population-based, real-world examination of practice patterns for colonoscopy in patients with acute LGIB in the United States. Despite the fact that current guidelines recommend that colonoscopy be performed within 24 hours in patients admitted with LGIB [6, 8], we found that as of 2014 , in a real-world setting, only $45 \%$ of patients had this recommended management. We recognize that there may be multiple potential explanations for this, including unavail- 
- Table 4 Factors affecting hospitalization costs in patients with LGIB who underwent colonoscopy.

\begin{tabular}{|c|c|c|c|c|}
\hline Variable & Odds Ratio & Lower $95 \% \mathrm{Cl}$ & Upper $95 \% \mathrm{Cl}$ & $P$ value \\
\hline Late colonoscopy without EGD & \multicolumn{4}{|l|}{ Referent } \\
\hline Early colonoscopy (log(LOS)-adjusted) & 0.88 & 0.87 & 0.88 & $<0.001$ \\
\hline Early colonoscopy [exact-matched] ${ }^{1}$ & 0.87 & 0.85 & 0.88 & $<0.001$ \\
\hline No EGD ${ }^{\dagger}$ & \multicolumn{4}{|l|}{ Referent } \\
\hline Early EGD & 0.99 & 0.98 & 0.99 & 0.04 \\
\hline Late EGD & 0.93 & 0.92 & 0.94 & $<0.001$ \\
\hline Age (per decade-increase) & 0.99 & 0.99 & 0.99 & $<0.001$ \\
\hline LOS (log-transformed) ${ }^{2}$ & 1.47 & 1.45 & 1.49 & $<0.001$ \\
\hline Weekend admission & 1.01 & 1.005 & 1.014 & $<0.001$ \\
\hline $\mathrm{CCI}^{\dagger}$ & 1.022 & 1.021 & 1.023 & $<0.001$ \\
\hline AKI & 1.12 & 1.115 & 1.133 & $<0.001$ \\
\hline Colectomy/colostomy in same admission & 1.93 & 1.89 & 1.97 & $<0.001$ \\
\hline ICU admission & 1.58 & 1.52 & 1.63 & $<0.001$ \\
\hline PRBC transfusion & 1.03 & 1.026 & 1.04 & $<0.001$ \\
\hline \multicolumn{5}{|l|}{ Hospital region } \\
\hline Northeast & \multicolumn{4}{|l|}{ Referent } \\
\hline Midwest & 0.98 & 0.96 & 0.99 & 0.009 \\
\hline South & 0.86 & 0.85 & 0.88 & $<0.001$ \\
\hline West & 1.14 & 1.12 & 1.17 & $<0.001$ \\
\hline \multicolumn{5}{|l|}{ Hospital location/teaching status } \\
\hline Rural & \multicolumn{4}{|l|}{ Referent } \\
\hline Urban non-teaching & 0.94 & 0.93 & 0.96 & $<0.001$ \\
\hline Urban teaching & 0.93 & 0.92 & 0.95 & $<0.001$ \\
\hline \multicolumn{5}{|l|}{ Hospital bed size } \\
\hline Small & \multicolumn{4}{|l|}{ Referent } \\
\hline Medium & 0.94 & 0.93 & 0.96 & $<0.001$ \\
\hline Large & 0.90 & 0.89 & 0.92 & $<0.001$ \\
\hline \multicolumn{5}{|c|}{$\begin{array}{l}\text { Note: The model was fitted to the log of hospitalization costs. } \\
\text { Analysis } N=751,552 \text {. Geometric mean hospitalization costs: } \$ 7,718 \text { USD. } \\
\text { Odds ratios reflect \% change over the geometric mean hospitalization costs. } \\
\text { LGIB, lower gastrointestinal bleeding; CI, confidence Interval; EGD, esophagogastroduodenoscopy; LOS, length of stay; CCI, Charlson Comorbidity Index; ICU, in- } \\
\text { tensive care unit. } \\
\text { These results are derived from multivariable analysis using mixed-effects log-transformed linear regression. } \\
1 \text { Exact-matched analysis on variables marked } \\
2 \text { in this Table as well as same hospital-year, yielding } 13,662 \text { matched observations (early colonoscopies } n=6,940) \text {, among 5,127 matching strata. Log(LOS), colect- } \\
\text { omy/colostomy, and ICU admission were added as confounders in the post-matching regression model. }\end{array}$} \\
\hline
\end{tabular}

ability of robust randomized data showing improved outcomes, difficulty with medical stabilization, and practicality of administering urgent bowel prep older population with multiple comorbid conditions. We did not find any significant trend toward performance of EC in LGIB patients. However, we noticed an increase in hospital admission for LGIB over the years. A study from Spain looking at data from 10 different hospital also reported increased LGIB admissions [4]. One potential reason for increasing LGIB admissions could be secondary to the increase in the aging population [24] and may be an increase in use of antithrombotics and/or anticoagulants, particularly in elderly patients with multiple comorbidities (who are more prone to LGIB).

The possibility that earlier endoscopic intervention can lead to rapid identification and control of culprit lesion bleeding and consequently could lead to reduced mortality in patients with 
Table 5 Factors affecting length of stay in patients with LGIB who underwent colonoscopy.

\begin{tabular}{|c|c|c|c|c|}
\hline Variable & Time Ratio & Lower $95 \% \mathrm{Cl}$ & Upper $95 \%$ Cl & $P$ value \\
\hline Late colonoscopy & \multicolumn{4}{|l|}{ Referent } \\
\hline Early Colonoscopy $(<24 \mathrm{~h})$ & 0.68 & 0.68 & 0.68 & $<0.001$ \\
\hline Early Colonoscopy $(<24 \mathrm{~h})$ [exact-matched] ${ }^{1}$ & 0.64 & 0.63 & 0.65 & $<0.001$ \\
\hline No EGD & \multicolumn{4}{|l|}{ Referent } \\
\hline Early EGD & 0.87 & 0.87 & 0.88 & $<0.001$ \\
\hline Late EGD & 0.96 & 0.96 & 0.97 & $<0.001$ \\
\hline Age (per decade-increase) & 1.05 & 1.048 & 1.053 & $<0.001$ \\
\hline Female & 1.05 & 1.045 & 1.056 & $<0.001$ \\
\hline Weekend admission & 0.99 & 0.980 & 0.991 & $<0.001$ \\
\hline $\mathrm{CCl}$ & 1.04 & 1.036 & 1.039 & $<0.001$ \\
\hline AKI & 1.20 & 1.19 & 1.21 & $<0.001$ \\
\hline Colectomy/colostomy in same admission & 2.38 & 2.34 & 2.43 & $<0.001$ \\
\hline ICU admission & 1.67 & 1.59 & 1.75 & $<0.001$ \\
\hline PRBC transfusion & 1.06 & 1.05 & 1.07 & $<0.001$ \\
\hline \multicolumn{5}{|l|}{ Insurance } \\
\hline Medicare & \multicolumn{4}{|l|}{ Referent } \\
\hline Medicaid & 1.04 & 1.03 & 1.05 & $<0.001$ \\
\hline Private & 0.95 & 0.94 & 0.95 & $<0.001$ \\
\hline Self-pay & 0.98 & 0.96 & 0.99 & 0.006 \\
\hline No charge & 1.00 & 0.96 & 1.04 & 0.808 \\
\hline Other & 1.00 & 0.98 & 1.02 & 0.709 \\
\hline \multicolumn{5}{|l|}{ Hospital region } \\
\hline Northeast & \multicolumn{4}{|l|}{ Referent } \\
\hline Midwest & 0.94 & 0.93 & 0.95 & $<0.001$ \\
\hline South & 0.96 & 0.95 & 0.97 & $<0.001$ \\
\hline West & 0.86 & 0.85 & 0.87 & $<0.001$ \\
\hline \multicolumn{5}{|l|}{ Hospital location/teaching status } \\
\hline Rural & \multicolumn{4}{|l|}{ Referent } \\
\hline Urban non-teaching & 1.05 & 1.04 & 1.07 & $<0.001$ \\
\hline Urban teaching & 1.06 & 1.05 & 1.08 & $<0.001$ \\
\hline \multicolumn{5}{|l|}{ Hospital bed size } \\
\hline Small & \multicolumn{4}{|l|}{ Referent } \\
\hline Medium & 1.04 & 1.03 & 1.05 & $<0.001$ \\
\hline Large & 1.08 & 1.06 & 1.09 & $<0.001$ \\
\hline \multicolumn{5}{|c|}{$\begin{array}{l}\text { Analysis } \mathrm{N}=794,455 \text {. Geometric mean length of stay: } 3.5 \text { days. } \\
\text { Area under the curve: } 0.774 \\
\text { Time ratios reflect \% change over the geometric mean length of stay } \\
\text { LGIB, lower gastrointestinal bleeding; Cl, confidence interval; EGD, esophagogastroduodenoscopy; CCI, Charlson Comorbidity Index; ICU, intensive care unit. } \\
\text { These results are derived from multivariable analysis using mixed-effects accelerated-failure-time (AFT) model with log-normal distribution. } \\
{ }^{1} \text { Exact-matched analysis on variables marked }{ }^{1} \text { in Table } 1 \text { and on number of diagnostic/operative procedures (in addition to colonoscopy), yielding 14,903 matched } \\
\text { observations (early colonoscopies } n=7,606 \text { ), among } 5,309 \text { matching strata. }\end{array}$} \\
\hline
\end{tabular}


LGIB makes timing of colonoscopy a critical point and area of interest in management of LGIB. Four RCTs $[10,11,13,14]$, six observational studies $[15,20,21,25-27]$ and seven meta-analyses [28-34] have been performed to evaluate this issue. One RCT showed that urgent colonoscopy led to identification of a definitive source of bleeding more frequently, but it did not lead to lower mortality [11]. Another RCT did not show any difference in clinical outcome with EC and it was prematurely terminated [10]. Similarly, observational studies [20,21] showed that EC provided no mortality benefit. Results from seven meta-analyses [28-34] have yielded mixed results, with four reporting no difference in diagnostic yield $[28,30,32,34]$ while three report increased diagnostic yield [29,31,33]; all showed no difference in rebleeding rate; all reported no difference in LOS except one [29]; no difference in transfusion requirement but one reported increased transfusion for EC [33]; all showed no difference in mortality rate. All these meta-analyses differed in the number of studies, and study designs included in quantitative synthesis along with their primary aim of study. Given the low mortality in LGIB, it is likely that RCTs with a very large sample size will be required to demonstrate a mortality benefit, if in fact there is one. We also acknowledge that because our study was non-randomized, it cannot definitively shed light on mortality benefit with EC.

The impact of timing of colonoscopy on clinical outcomes other than mortality is important in clinical management. In one study, rapid colonoscopy with endoscopic therapy within 12 hours of presentation in patients with severe hematochezia and diverticulosis resulted in reduced rebleeding rate and need for surgery [12]. One study reported lower transfusion requirement associated with early endoscopy [21] and an NIS-based study showed that EC reduced LOS and cost of hospitalization [15]. A meta-analysis showed a decreased LOS and fewer complications with early endoscopy [29]. A recent meta-analysis [32] that only included RCTs reported no change in LOS with EC. However, a statistical analysis was not performed to specifically evaluate this result. Another recent meta-analysis studied impact of timing on LOS for RCTs and observational studies separately: no difference in LOS was found for RCTs only, but when only observational studies were analyzed, LOS was statistically reduced [34]. In our study, we found that EC was associated with a substantially decreased hospital LOS (by 1.6 days) in comparison to late colonoscopy, consistent with several other studies [15, 20-23]. Therefore, we presume that EC may lead to early triage of patients and shorter wait time to get colonoscopy, and thus subsequently earlier discharge, which could explain the short LOS. As expected, reduced LOS ultimately translates into cost reduction and, as expected, our study showed a decrease in cost of hospitalization by $\$ 1,946$ with EC even after adjusting for LOS.

Patients who are admitted on weekends for LGIB represent a challenging group because in many hospitals, appropriate staffing to perform procedures such as colonoscopy is limited or unavailable. The impact of weekend admission on patients with UGIB has been variable in terms of mortality, LOS, and cost of hospitalization [35, 36]. A previous NIS-based study reported no difference in mortality but increased LOS with week- end admission in LGIB patients [15]. In our study, we also found that weekend admission did not affect mortality, LOS or cost of hospitalization compared to weekday admission. The LC group had more weekend admissions, implying that issues with logistics over the weekend may led to delays in colonoscopy and increased costs. Arguing against this possibility is the finding that weekend admission in our study was not associated with higher cost compared to weekday admission. Thus, our study does not support a "weekend effect."

In our multivariable regression analysis, increments in age and comorbidity were associated with an increase in mortality. Both of these variables are well-known risk factors for death and have been incorporated in risk stratification tools in UGIB [37-39]. Age $>70$ years and more than two comorbidities have been identified as independent predictors of mortality [37-39]. Our study reiterates impact of age and comorbidities in risk stratification for this patient population. Diverticular bleeding represents the largest proportion of LGIB cases [40]. After separating the diverticular bleeding and non-diverticular bleeding cohort, we compared the mortality rate between the two and found no statistically significant difference.

We recognize the limitations of our study. As with all population-based studies, the diagnosis of LGIB is reliant on accurate diagnostic coding. Our study utilized ICD-9 coding methodology similar to a prior population-based study in this area [15, 16]. In addition, given the nature of the NIS data, we were unable to evaluate rebleeding rates, a relevant clinical outcome in LGIB. Similarly, because of unavailability of Current Procedural Terminology codes and non-specific ICD-9 codes for endoscopic interventions, we were unable to ascertain whether an intervention was performed during the colonoscopy itself. Some results that are statistically significant on bivariate analysis may not be clinically significant (i.e., age between the EC and LC groups), which may suggest type II error. In addition, our study results are only applicable to patients admitted with acute LGIB in the United States. However, our study has several strengths and clinical applications. With this large NIS-based study encompassing data over 10 year, we studied the largest sample to date in this area, which is also free of biases that may be introduced in studies from small individual centers. This suggests that our results are likely generalizable across the entire US population.

\section{Conclusion}

In summary, our study demonstrated that practice patterns in terms of performance of EC have not changed over the last decade. Admissions with acute LGIB are on the rise with an improved all-cause inpatient mortality rate. Further, the data indicate that timing of colonoscopy - early or late - does not appear to influence overall inpatient mortality. However, the data indicated that EC was associated with decreased healthcare costs compared with LC and suggest that EC may lead to more efficient patient care. 
Competing interests

The authors declare that they have no conflict of interest.

\section{References}

[1] Strate LL, Gralnek IM. ACG Clinical Guideline: Management of patients with acute lower gastrointestinal bleeding. Am J Gastroenterol 2016; 111: 459-474

[2] Laine L, Yang H, Chang SC et al. Trends for incidence of hospitalization and death due to $\mathrm{GI}$ complications in the United States from 2001 to 2009. Am J Gastroenterol 2012; 107: 1190-1195 quiz 1196

[3] Peery AF, Crockett SD, Murphy CC et al. Burden and cost of gastrointestinal, liver, and pancreatic diseases in the United States: Update 2018. Gastroenterology 2018; 156: 254-272

[4] Lanas A, Garcia-Rodriguez LA, Polo-Tomas M et al. Time trends and impact of upper and lower gastrointestinal bleeding and perforation in clinical practice. Am J Gastroenterol 2009; 104: 1633-1641

[5] Lhewa DY, Strate LL. Pros and cons of colonoscopy in management of acute lower gastrointestinal bleeding. World J Gastroenterol 2012; 18: $1185-1190$

[6] Strate LL, Gralnek IM. ACG Clinical Guideline: Management of patients with acute lower gastrointestinal bleeding. Am J Gastroenterol 2016; 111: 755

[7] Soetikno R, Ishii N, Kolb JM et al. The role of endoscopic hemostasis therapy in acute lower gastrointestinal hemorrhage. Gastrointest Endosc Clin N Am 2018; 28: 391-408

[8] Pasha SF, Shergill A, Acosta RD et al. The role of endoscopy in the patient with lower GI bleeding. Gastrointest Endosc 2014; 79: 875-885

[9] Davila RE, Rajan E, Adler DG et al. ASGE Guideline: the role of endoscopy in the patient with lower-GI bleeding. Gastrointest Endosc 2005; 62: 656-660

[10] Laine L, Shah A. Randomized trial of urgent vs. elective colonoscopy in patients hospitalized with lower GI bleeding. Am J Gastroenterol 2010; 105: 2636-2641 quiz 2642

[11] Green BT, Rockey DC, Portwood G et al. Urgent colonoscopy for evaluation and management of acute lower gastrointestinal hemorrhage: a randomized controlled trial. Am J Gastroenterol 2005; 100: 23952402

[12] Jensen DM, Machicado GA, Jutabha R et al. Urgent colonoscopy for the diagnosis and treatment of severe diverticular hemorrhage. N Engl J Med 2000; 342: 78-82

[13] van Rongen I, Thomassen BJW, Perk LE. Early versus standard colonoscopy: a randomized controlled trial in patients with acute lower gastrointestinal bleeding: results of the BLEED study. J Clin Gastroenterol 2019; 53: 591-598

[14] Niikura R, Nagata N, Yamada A et al. Efficacy and safety of early vs elective colonoscopy for acute lower gastrointestinal bleeding. Gastroenterology 2020; 158: 168-175 e166

[15] Navaneethan U, Njei B, Venkatesh PG et al. Timing of colonoscopy and outcomes in patients with lower GI bleeding: a nationwide population-based study. Gastrointest Endosc 2014; 79: 297-306 e212

[16] Strate LL, Ayanian JZ, Kotler G et al. Risk factors for mortality in lower intestinal bleeding. Clin Gastroenterol Hepatol 2008; 6: 1004-1010 quiz 1955

[17] Agency for Healthcare Research and Quality R, MD. HCUP NIS Description of Data Elements. Healthcare Cost and Utilization Project (HCUP). 2008: www.hcup-us.ahrq.gov/db/vars/prdayn/nisnote.jsp

[18] Quan H, Li B, Couris CM et al. Updating and validating the Charlson Comorbidity Index and score for risk adjustment in hospital discharge abstracts using data from six countries. Am J Epidemiol 2011; 173: 676-682

[19] Quan H, Sundararajan V, Halfon P et al. Coding algorithms for defining comorbidities in ICD-9-CM and ICD-10 administrative data. Med Care 2005; 43: 1130-1139

[20] Nagata N, Niikura R, Sakurai T et al. Safety and effectiveness of early colonoscopy in management of acute lower gastrointestinal bleeding on the basis of propensity score matching analysis. Clin Gastroenterol Hepatol 2016; 14: 558-564

[21] Nigam N, Patel P, Sengupta N. Outcomes of early versus delayed colonoscopy in lower gastrointestinal bleeding using a hospital administrative database. J Clin Gastroenterol 2018; 52: 721-725

[22] Schmulewitz N, Fisher DA, Rockey DC. Early colonoscopy for acute lower $\mathrm{Gl}$ bleeding predicts shorter hospital stay: a retrospective study of experience in a single center. Gastrointest Endosc 2003; 58: 841846

[23] Strate LL, Syngal S. Timing of colonoscopy: impact on length of hospital stay in patients with acute lower intestinal bleeding. Am J Gastroenterol 2003; 98: 317-322

[24] He WGD, Kowa P. An Aging World. 2015: https://www.census.gov/ content/dam/Census/library/publications/2016/demo/p95-16-1.pdf

[25] Albeldawi M, Ha D, Mehta P et al. Utility of urgent colonoscopy in acute lower gastro-intestinal bleeding: a single-center experience. Gastroenterol Rep (Oxf) 2014; 2: 300-305

[26] Saraireh H, Tayyem O, Siddiqui MT et al. Early colonoscopy in patients with acute diverticular bleeding is associated with improvement in healthcare-resource utilization. Gastroenterol Rep (Oxf) 2019; 7: $115-120$

[27] Kim JH, Chun J, Lee $\mathrm{C}$ et al. Early versus late bedside endoscopy for gastrointestinal bleeding in critically ill patients. Korean J Intern Med 2018; 33: 304-312

[28] Kouanda AM, Somsouk M, Sewell JL et al. Urgent colonoscopy in patients with lower $\mathrm{Gl}$ bleeding: a systematic review and meta-analysis. Gastrointest Endosc 2017; 86: 107-117 e101

[29] Roshan Afshar I, Sadr MS, Strate LL et al. The role of early colonoscopy in patients presenting with acute lower gastrointestinal bleeding: a systematic review and meta-analysis. Therapeutic Advances in Gastroenterology 2018; 11: 1756283X18757184

[30] Seth A, Khan MA, Nollan R et al. Does urgent colonoscopy improve outcomes in the management of lower gastrointestinal bleeding? Am J Med Sci 2017; 353: 298-306

[31] Sengupta N, Tapper EB, Feuerstein JD. Early versus delayed colonoscopy in hospitalized patients with lower gastrointestinal bleeding: a meta-analysis. J Clin Gastroenterol 2017; 51: 352-359

[32] Tsay C, Shung D, Stemmer Frumento K et al. Early colonoscopy does not improve outcomes of patients with lower gastrointestinal bleeding: systemativ review of randomized trials. Clin Gastroenterol Hepatol 2020; 18: 1696-1703 e1692

[33] Oakland K, Isherwood J, Lahiff C et al. Diagnostic and therapeutic treatment modalities for acute lower gastrointestinal bleeding: a systematic review. Endosc Int Open 2017; 5: E959-E973

[34] Kherad O, Restellini S, Almadi M et al. Systematic review with metaanalysis: limited benefits from early colonoscopy in acute lower gastrointestinal bleeding. Aliment Pharmacol Ther 2020; 52: 774-788

[35] Ananthakrishnan AN, McGinley EL, Saeian K. Outcomes of weekend admissions for upper gastrointestinal hemorrhage: a nationwide analysis. Clin Gastroenterol Hepatol 2009; 7: 296-302e291

[36] Abougergi MS, Travis AC, Saltzman JR. Impact of day of admission on mortality and other outcomes in upper $\mathrm{Gl}$ hemorrhage: a nationwide analysis. Gastrointest Endosc 2014; 80: 228-235

[37] Laursen SB, Oakland K, Laine L et al. ABC score: a new risk score that accurately predicts mortality in acute upper and lower gastrointesti- 
nal bleeding: an international multicentre study. Gut 2020: doi:10.1136/gutjnl-2019-320002

[38] Oakland K, Kothiwale S, Forehand T et al. External validation of the Oakland score to assess safe hospital discharge among adult patients with acute lower gastrointestinal bleeding in the US. JAMA Netw Open 2020; 3: e209630
[39] Sengupta N, Tapper EB. Derivation and internal validation of a clinical prediction tool for 30-day mortality in lower gastrointestinal bleeding. Am J Med 2017; 130: 601 e601-601 e608

[40] Ghassemi KA, Jensen DM. Lower GI bleeding: epidemiology and management. Curr Gastroenterol Rep 2013; 15: 333 